for impacting the observed response rates, while the effect upon limiting the pool of eligible subjects is often poorly understood. Unnecessarily narrow criteria may limit the generalizability of trial results to real world patient populations. Conversely, certain patient characteristics may be associated with high or low probabilities of response to active treatment or to placebo.

Objectives: To assess the impact of applying a range of different exclusion criteria on the proportion eligible subjects, and on the observed treatment response rates, in a large real-world patient population.

Methods: Data on RA characteristics, demographics, and co-morbidities among RA patients were identified by linking the Swedish Rheumatology register (SRQ) to the nationwide and virtually complete Swedish census and healthcare registries. Representing an early RA trial scenario, we identified patients starting methotrexate monotherapy as first ever DMARD $(\mathrm{N}=8981)$ between 2007 and 2016. The cohort was assessed overall and stratified by baseline DAS28. Treatment outcome was defined as the proportions reaching (1) EULAR Good Response and (2) Low Disease Activity, and the change in (3) $\mathrm{HAQ}$ and (4) CDAl at 3 and 6 months. Exclusions were made based on age, baseline disease activity, sex, RF, predefined comorbidities, degree of healthcare utilization history, education, and taxed income level (cut-offs for continuous variables were based on distributions from recent clinical trials of tofacitinib). In total, 165 different definitions of exclusion criteria were evaluated.

Results: Within the entire cohort, $50 \%$ of patients achieved EULAR DAS28 good response at 3 months. Exclusions based on age, sex, RF status, or duration of RA symptoms before RA diagnosis generally lead to large reductions in number of eligible subjects but did not appreciably affect $(<5 \%$ change) the proportion of EULAR good responders. Exclusions based on HAQ had a noticeable drop in the proportion of EULAR good response $(-10 \%$, see Figure), although exclusions above $80 \%$ was necessary to observe an effect. A similar pattern was observed for TJC, patient's global health and ESR but exclusions based on SJC or CRP did not impact EULAR response.

Exclusions of specific comorbidities, such as history of myocardial infarction, history of joint replacement, generally had no or modest impact on the observed response rate. Exclusions based on health care use (total number of drugs, hospitalizations), affected response rates (+/- around $5 \%$ ), while exclusions based on educational level, work ability, or income did not.

The impact of exclusions was very similar on the 3 and 6 month responses.

Conclusion: Exclusions in a range of criteria commonly used in clinical trials had only a modest impact on observed treatment outcome, while impacting on the enrollment pool - sometimes dramatically. More extreme restrictions (excluding well above half the potentially eligible patients) were generally necessary to shift the proportion EULAR Good Response by more than 5 percentage points. This should not be a surprise given the lack of identified strong predictors of treatment outcome in RA, but may raise the question of whether the lowered generalizability and impacts on enrollment rates caused by strict inclusion criteria in clinical trials is warranted by the aspiration to increase chances of demonstrating clinically meaningful effects.

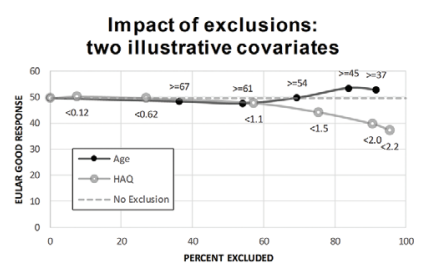

Abstract THU0076- Figure 1

Disclosure of Interests: Thomas Frisell: None declared, Scott Jelinsky Shareholder of: Pfizer, Employee of: Pfizer, Mark Peterson Shareholder of: Pfizer, Employee of: Pfizer, Johan Askling Grant/research support from: Karolinska Institutet (JA) has or has had research agreements with the following pharmaceutical companies, mainly in the context of the ATRIS national safety monitoring programme for rheumatology biologicals: Abbvie, BMS, MSD, Eli Lilly, Pfizer, Roche, Samsung Bioepis, and UCB., Consultant for: Karolinska Institutet has received remuneration for JA participating in ad boards arranged by Lilly, Novartis, and Pfizer.

DOI: 10.1136/annrheumdis-2019-eular.1022

\section{THU0077 RHEUMATOID ARTHRITIS AND ANKYLOSING SPONDYLITIS CLINICAL TRIALS: HOW TO ENCOURAGE PATIENTPARTICIPATION? RESULTS FROM A REAL- WORLD STUDY CONDUCTED VIA CARENITY, A WORLDWIDE ONLINE PATIENT COMMUNITY}

remi gauchoux, Anthony Boisbouvier, Roman Dos Santos, Lise Radoszycki. CARENITY, Paris, France

Background: Involving patients affected with rheumatoid arthritis or anky losing spondylitis in clinical trials may be highly challenging: in a worldwide context of competitive rheumatoid arthritis and ankylosing spondylitis clinical research, patient engagement is key to optimize medical research and increase participation rates.

Objectives: The aim of this study was to identify patients' motivations for and hindrances to joining a trail in order to increase participation rate by implementing tailored services and information.

Methods: An online questionnaire was submitted from August 2017 to October 2017 to Carenity's French members affected with rheumatoid arthritis or ankylosing spondylitis.

Results: 136 patients affected with rheumatoid arthritis $(n=55,40 \%$, mean age $=55 \mathrm{y} / 0,71 \%$ of women) or ankylosing spondylitis ( $\mathrm{n}=81$, $60 \%$, mean age $=48 \mathrm{y} / \mathrm{o}, 80 \%$ of women) participated in the study. 10 patients $(7 \%)$ already participated in a clinical trial.

Non-participants $(n=126)$ exposed the incentives that may increase participation rate: reimbursing the expenses related to the clinical trial can improve their motivation (median weight on motivation to join a trial = $10 / 10$ ) as well as a better medical follow-up (8.0/10) or leveraging the trial's investigator notoriety (8.0/10). Patients' healthcare practitioners could be leveraged as well: their favorable opinion turns patients on to clinical trials (7.0/10). On the contrary, a doctor's negative opinion may strongly deter patients from joining a trial (median weight on reluctance to join a trial $=8 \cdot 0 / 10)$. Communication should aim at reassuring them about side effects $(9.0 / 10)$, potential risks for their health $(8.0 / 10)$ and more generally the risk that the new treatment would not be better than their current one (8.0/10). Logistic challenges should also be taken into account: the trip between patients' home and the site of the clinical trial is perceived as an important burden (8.0/10).

Implementing tailored services and information would be an efficient way to reassure patients and increase their willingness to participate. $81 \%$ of nonparticipants would be enticed to participate if they had access to a website to exchange with healthcare professionals or to follow the results of the trial. $79 \%$ would be interested in participants' testimonials and $74 \%$ in a 24-hour phone helpline. Spreading and clarifying information before the trial is also critical: $6 / 10$ patients who participated in a clinical trial did not understand clearly if they will have out-of-pocket costs and $5 / 10$ patients were also unclear on the terms and conditions for leaving the clinical trial. Informing patients via convenient channels is key: $88 \%$ of participants and non-participants think that an online patient community is a relevant medium to convey information about clinical trials and $81 \%$ of non-participants would like to have access to an information brochure about the trial process.

Conclusion: This real-world study allowed to identify concrete levers to reassure patients affected with rheumatoid arthritis or ankylosing spondylitis about the benefits/constraints balance and to clarify information at each stage of the trial (before, during, after), which is essential to accelerate clinical trial recruitment.

Disclosure of Interests: None declared

DOI: 10.1136/annrheumdis-2019-eular.6922

\section{THU0078 \\ SAFETY PROFILE OF BARICITINIB FOR THE TREATMENT OF RHEUMATOID ARTHRITIS UP TO 7 YEARS: AN UPDATED INTEGRATED SAFETY ANALYSIS}

Mark C. Genovese ${ }^{1}$, Josef S. Smolen ${ }^{2}$, Tsutomu Takeuchi ${ }^{3}$, Gerd

Rüdiger Burmester ${ }^{4}$, Dennis Brinker ${ }^{5}$, Terence Rooney ${ }^{5}$, Jinglin Zhong ${ }^{6}$, Daojun $\mathrm{Mo}^{5}$. Chadi Saifan ${ }^{5}$, Anabela Cardoso ${ }^{5}$, Maher Issa ${ }^{5}$, Wen-Shuo Wu ${ }^{5}$, Kevin Winthrop? ${ }^{1}$ Stanford University Medical Center, Palo Alto, United States of America; ${ }^{2}$ Medical University of Vienna, Vienna, Austria; ${ }^{3}$ Keio University, Tokyo, Japan; ${ }^{4}$ Charité - University Medicine Berlin, Berlin, Germany; ${ }^{5}$ Eli Lilly and Company, Indianapolis, United States of America; ${ }^{6} I Q V I A$, Rockville, United States of America; ${ }^{7}$ Oregon Health Sciences University, Portland, United States of America

Background: Baricitinib (bari), is an oral, selective inhibitor of Janus kinase (JAK) 1/JAK 2, to treat moderately to severely active RA in adults. Objectives: To update bari's safety profile with data from an additional Phase (Ph) 3 trial and on-going long-term extension (LTE) study. Methods: Long-term safety of once-daily bari was evaluated in the AllBari-RA dataset: all patients (pts) exposed to any bari dose from 9 